\title{
Induction of B7-1 in podocytes is associated with nephrotic syndrome
}

\author{
Jochen Reiser, ${ }^{1}$ Gero von Gersdorff, ${ }^{1}$ Martin Loos, ${ }^{1}$ Jun Oh, ${ }^{1}$ Katsuhiko Asanuma, ${ }^{1}$ Laura Giardino, \\ Maria Pia Rastaldi, ${ }^{2}$ Novella Calvaresi, ${ }^{2}$ Haruko Watanabe, ${ }^{3}$ Karin Schwarz, ${ }^{1}$ Christian Faul, ${ }^{1,4}$ \\ Matthias Kretzler, ${ }^{5}$ Anne Davidson,1,6 Hikaru Sugimoto,7 Raghu Kalluri,7 Arlene H. Sharpe,8 \\ Jordan A. Kreidberg, ${ }^{9}$ and Peter Mundel1,4
}

${ }^{1}$ Department of Medicine, Albert Einstein College of Medicine, New York, New York, USA. ${ }^{2}$ Renal Research Association, Renal Immunopathology Center, San Carlo Borromeo Hospital, Milan, Italy. ${ }^{3}$ Department of Pediatrics, Tokyo Metropolitan Toshima Hospital, Tokyo, Japan. ${ }^{4}$ Department of Anatomy and Structural Biology, Albert Einstein College of Medicine, New York, New York, USA. ${ }^{5}$ Department of Medicine, Ludwig-Maximilians-Universität, Munich, Germany. ${ }^{6}$ Department of Microbiology and Immunology, Albert Einstein College of Medicine, New York, New York, USA. ${ }^{7}$ Center for Matrix Biology, Department of Medicine, Beth Israel Deaconess Medical Center and Harvard Medical School, Boston, Massachusetts, USA. ${ }^{8}$ Department of Pathology, Brigham and Women's Hospital and Harvard Medical School, Boston, Massachusetts, USA. 9Department of Medicine, Children's Hospital, and Department of Pediatrics, Harvard Medical School, Boston, Massachusetts, USA.

\begin{abstract}
Kidney podocytes and their slit diaphragms form the final barrier to urinary protein loss. This explains why podocyte injury is typically associated with nephrotic syndrome. The present study uncovered an unanticipated novel role for costimulatory molecule B7-1 in podocytes as an inducible modifier of glomerular permselectivity. B7-1 in podocytes was found in genetic, drug-induced, immune-mediated, and bacterial toxin-induced experimental kidney diseases with nephrotic syndrome. The clinical significance of our results is underscored by the observation that podocyte expression of B7-1 correlated with the severity of human lupus nephritis. In vivo, exposure to low-dose LPS rapidly upregulates B7-1 in podocytes of WT and SCID mice, leading to nephrotic-range proteinuria. Mice lacking B7-1 are protected from LPS-induced nephrotic syndrome, suggesting a link between podocyte B7-1 expression and proteinuria. LPS signaling through toll-like receptor-4 reorganized the podocyte actin cytoskeleton in vitro, and activation of B7-1 in cultured podocytes led to reorganization of vital slit diaphragm proteins. In summary, upregulation of B7-1 in podocytes may contribute to the pathogenesis of proteinuria by disrupting the glomerular filter and provides a novel molecular target to tackle proteinuric kidney diseases. Our findings suggest a novel function for B7-1 in danger signaling by nonimmune cells.
\end{abstract}

\section{Introduction}

The kidney glomerulus is a highly specialized structure that ensures selective ultrafiltration of plasma so that essential proteins are retained in the blood. The common denominator in a variety of kidney diseases, such as diabetes mellitus, focal segmental glomerulosclerosis, and systemic lupus erythematosus, is glomerular dysfunction (nephrotic syndrome), involving a massive loss of protein in the urine (proteinuria) (1). Kidney or systemic diseases that result in nephrotic syndrome are important causes of morbidity and mortality affecting both adults and children. Glomerular podocytes serve as the final barrier to urinary protein loss by the formation and maintenance of podocyte foot processes (FPs) and the interposed slit diaphragm (SD) (1). All forms of nephrotic syndrome are characterized by abnormalities in podocytes, including retraction (effacement) of podocyte FPs and/or molecular reorganization of the SD. FP effacement is a dynamic process that correlates closely with the development of proteinuria both in human disease and in experimental models. FP effacement requires a precise interplay of multiple cellular functions, including struc-

Nonstandard abbreviations used: foot process (FP); glomerular basement membrane (GBM); puromycin aminonucleoside (PAN); slit diaphragm (SD); toll-like receptor (TLR)

Conflict of interest: The authors have declared that no conflict of interest exists.

Citation for this article: J. Clin. Invest. 113:1390-1397 (2004).

doi:10.1172/JCI200420402. tural alterations of the cytoskeleton, movement of FPs over the basement membrane, and reconstruction of the SD (1). From a clinical perspective, it is important to recognize that early structural changes in podocytes such as substructural alterations in SD composition or FP effacement have to be reversed within a certain period of time to prevent development of severe and progressive glomerular damage (2). Recent years have been extremely fruitful in providing an extensive amount of information on the molecular composition of the SD and its anchoring structures, which form multiple interactions and provide signaling platforms. The discovery of several novel podocyte proteins and their mutation analysis, including nephrin (3), CD2AP (4), $\alpha$-actinin-4 (5), podocin (6), and neph1 (7), have shed light on the pathogenesis of proteinuria and emphasized the critical role of the podocyte and the SD in maintaining the function of the glomerular filtration barrier.

Podocyte FPs form a scaffolding around the capillary loops that are anchored to the glomerular basement membrane $(\mathrm{GBM})$ via $\alpha 3 \beta 1$ integrin $(8)$ and $\alpha$-/ $\beta$-dystroglycans $(9,10)$. $\alpha 3 \beta 1$ integrin represents a receptor for certain isoforms of laminin, including laminin-5 in the epidermal basement membrane and laminin-10/11 in the GBM (11). In podocytes, $\alpha 3 \beta 1$ integrin is expressed at the basal side of FPs. Podocytes of $\alpha 3$ integrin-deficient $\left(\alpha 3^{-/-}\right)$mice bear a strong morphological resemblance to podocytes in congenital nephrotic syndrome and other pathological conditions with FP effacement $(11,12)$. The $\alpha 3$ integrin deletion therefore represents a genetic model 
system that allows the analysis of the molecular basis of FP assembly and dynamics.

B7-1, also termed CD80, is a transmembrane protein normally expressed on the surface of B cells and other APCs (13-15). On APCs, B7-1 acts as a costimulatory molecule through binding to its cognate receptors CD28 and CTLA-4 on T cells (13-15). Little is known about B7-1 expression by non-bone marrow-derived cells. In human keratinocytes, B7-1 gene expression is upregulated by allergens and irritants (16). In P815 mastocytoma tumor cells, B7-1 gene expression is induced by oxidative stress (17), which is reminiscent of the induction of B7-1 in podocytes by puromycin, described below. In the kidney, B7-1 can be induced in glomerular endothelial cells by warm ischemia/reperfusion injury in rats (18).

The innate immune system senses pathogen-associated molecular motifs through toll-like receptors (TLRs), thereby initiating an immunological response (19). In APCs, LPS is the strongest endogenous inducer of B7-1 expression through TLR-4 signaling (20). Here we show that danger signaling occurs also in non-bone marrow-derived glomerular podocytes and underlies a dramatic physiological response. Podocytes sense LPS by TLR-4, which triggers the reorganization of the kidney-filtration apparatus, leading to podocyte FP effacement and proteinuria. This response requires the induction of costimulatory molecule $\mathrm{B} 7-1$, since proteinuria was abrogated in B7-1/- mice. We discuss a novel function for B7-1 in the danger signaling of nonimmune cells.

\section{Methods}

Cell culture. WT podocytes were cultured as described before (21). We generated conditionally immortalized $\alpha 3^{-/-}$podocyte cell lines by intercrossing $\alpha 3$ integrin heterozygous mice (12) with the ImmortoMouse (Charles River Laboratories Inc., Wilmington, Massachusetts, USA), which carries a temperature-sensitive $\mathrm{T}$ antigen as transgene (22). Glomeruli were isolated from kidneys of E18 homozygous embryos, and eight $\alpha 3^{-/-}$clonal podocyte cell lines were obtained by limiting dilution. To show that observed changes in podocyte gene expression and function were due to the absence of $\alpha 3$ integrin, three $\alpha 3^{-/-}$clones were stably transfected with human $\alpha 3$ integrin cDNA as described before for $\alpha 3^{-/-}$collecting duct epithelial cells (23).

Differential display PCR. Total RNA was isolated from differentiated WT and $\alpha 3^{-/-}$podocytes that had been grown under nonpermissive conditions for 14 days using the RNeasy kit (QIAGEN Inc., Valencia, California, USA). After DNase treatment, reverse transcription was carried out with three different anchored synthetic oligonucleotide primers from the RNAimage kit (GenHunter, Nashville, Tennessee, USA). Differential display PCR reactions were performed in duplicate using 96 different primer combinations exactly as described before (24). Differentially expressed PCR products were cloned into the PCR2 vector using the TA cloning kit (Invitrogen, Carlsbad, California, USA) and sequenced. Sequence analyses and database searches were done with the software program package HUSAR (Heidelberg Unix Sequence Analysis Resources; German Cancer Research Center, Heidelberg, Germany). The following primers specific for B7-1, yielding a 172-bp product, were used: sense, $5^{\prime}$-TGTATGCCCAGGAAACAGGT-3'; antisense, 5'-AGCCCGATCACCAC-TGATTA-3'.

Antibodies. The following protein-specific primary antibodies were used: anti-B7-1 (16-10A1; Pharmingen, San Diego, California, USA), anti-B7-1 (R\&D Systems Inc., Minneapolis, Minnesota,
USA), and mouse monoclonal anti-synaptopodin (25). Rabbit was a gift from L. Holzman (University of Michigan, Ann Arbor, Michigan, USA); rabbit polyclonal anti-nephrin CD2AP was a gift from A. Shaw (Washington University, St. Louis, Missouri, USA); polyclonal anti-ZO-1 was from Zymed Laboratories Inc. (South San Francisco, California, USA).

Immunocytochemistry. Human and murine kidneys were harvested and snap-frozen according to standard protocols and fixed after sectioning in ice-cold acetone for 10 minutes. For immunofluorescent labeling, sections were washed once with PBS and incubated with blocking solution (2\% FCS, $2 \%$ BSA, $0.2 \%$ fish gelatin) for 30 minutes at room temperature before further incubation with one of the primary antibodies for 1 hour at room temperature. For double labeling, sections were washed three times with PBS for 5 minutes, and the second primary antibody was applied for 60 minutes. Antigen-antibody complexes were visualized with secondary antibodies (Rockland Inc., Gilbertsville, Pennsylvania, USA) conjugated with fluorochrome (Cy2 or Cy3). Pictures were captured with a Spot cooled CCD camera (Diagnostic Instruments, Sterling Heights, Michigan, USA) and processed with Adobe Photoshop 5.0 software (Adobe Systems Inc., San Jose, California, USA). Confocal microscopy and processing of micrographs were performed as reported before (25).

LPS treatment of cultured podocytes. Differentiated podocytes were exposed to different doses of LPS $(1-50 \mu \mathrm{g} / \mathrm{ml})$ for 3.5 to 72 hours. RT-PCR for B7-1 was done as described above, and changes in the organization of the actin cytoskeleton were assessed by phalloidin staining as described before (26). Each set of experiments was repeated at least five times.

Nephrin ${ }^{-1-}$ mice. The generation and functional characterization of nephrin ${ }^{-/}$mice has been reported before (27). For the present study, kidneys were harvested from 1-day-old nephrin ${ }^{-/-}$and WT littermate animals, snap-frozen in liquid nitrogen, and processed for immunohistochemistry as described above.

LPS-induced FP effacement and proteinuria. All animal studies were approved by the Albert Einstein College of Medicine Animal Institute Committee. B7-1/- mice were generated and backcrossed onto a BALB/c background (>12 generations) as previously described (28). SCID mice (29) on a BALB/c background as well as WT 129 and C57BL/6 mice were obtained from The Jackson Laboratory (Bar Harbor, Maine, USA). The base-line urine protein excretion of 4- to 6-week-old female $129(n=10)$, C57BL/6 $(n=25), \mathrm{BALB} / \mathrm{c}(n=13), \mathrm{B} 7-1^{-/-}(n=5)$, and SCID $(n=6)$ mice was analyzed by urine dipstick (Albustix; Bayer, Tarrytown, New York, USA) before animals were injected intraperitoneally with either $200 \mu \mathrm{g}$ LPS ( $1 \mathrm{mg} / \mathrm{ml}$ in sterile LPS-free PBS) in a total volume of $200 \mu \mathrm{l}$, or equal volumes of sterile LPS-free PBS (PBS: BALB/c WT, $n=5$; B7-1//-, $n=3$; SCID, $n=3$ ). Twenty-four hours after injection, urinary protein excretion was measured again, and kidneys were harvested and processed for $\mathrm{H} \& \mathrm{E}$ and immunofluorescence staining. FP effacement was assessed by transmission electron microscopy according to our published protocols (30). In a subset of C57BL mice $(n=5)$, urine was collected before and 24 hours after LPS injection and analyzed by dipstick. From the same urines we determined the protein/creatinine ratio with Albuwell $\mathrm{M}$ and Creatinine Companion protein/ creatinine ELISA kits (Exocell Inc., Philadelphia, Pennsylvania, USA), respectively. All measurements were performed in duplicate. The following results were obtained: protein/creatinine 

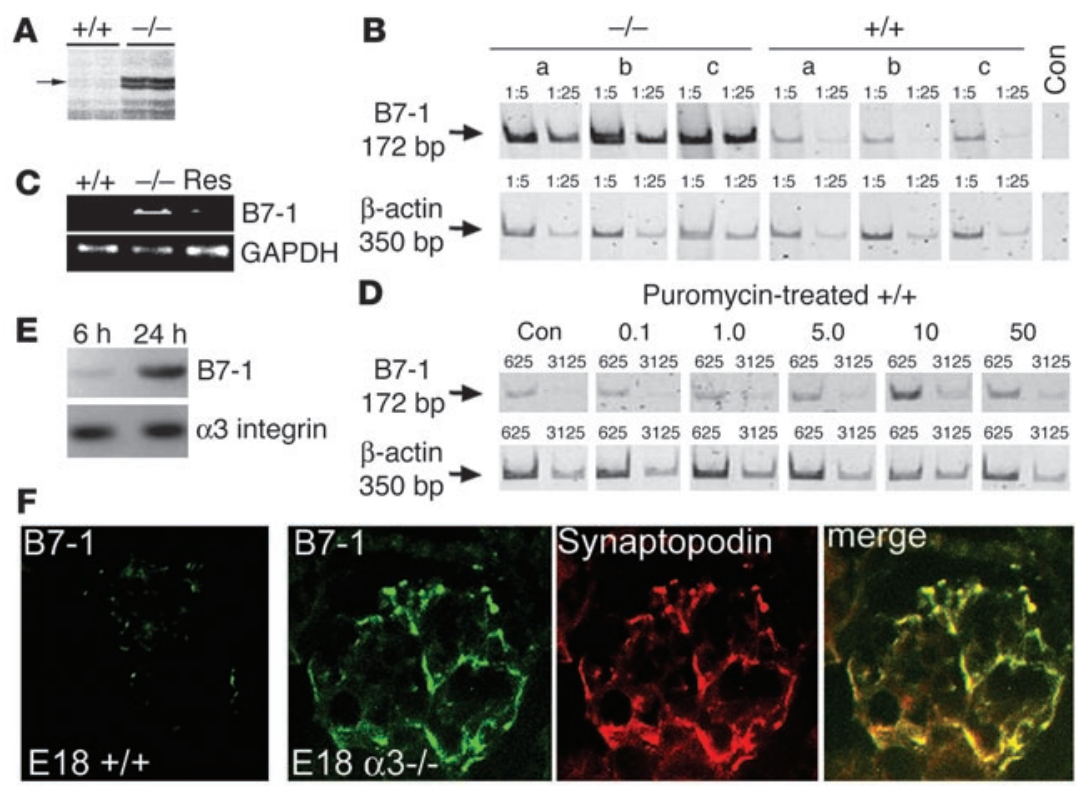

\section{Figure 1}

B7-1 expression in podocytes. (A) Differential display PCR of WT (+/+) and $\alpha 3^{-/-}(-/-)$podocytes. The arrow indicates the position of B7-1. (B) Induction of B7-1 mRNA expression in $\alpha 3^{-/}$podocytes. RT-PCR from three independent samples $(\mathrm{a}-\mathrm{C})$ on serial dilution (1:5 to $1: 25)$ of cDNA standardized for $\beta$-actin. Con, $\mathrm{H}_{2} \mathrm{O}$ control. (C) Rescue (Res) of $\alpha 3^{-/-}$podocytes by stable transfection with human $\alpha 3$ integrin restored B7-1 expression to WT levels. RT-PCR for GAPDH shows equal RNA concentrations. (D) Dose-dependent increase of B7-1 mRNA in WT podocytes in response to puromycin $(0.1,1,5,10$, and $50 \mu \mathrm{g} / \mathrm{ml})$. (E) Time course of B7-1 and $\alpha 3$ integrin mRNA expression in puromycin aminonucleoside-treated (PAN-treated) WT podocytes. As early as 6 and 24 hours, B7-1 is induced by PAN, whereas $\alpha 3$ integrin levels remain unchanged. (F) B7-1 is not expressed in E18 WT kidneys (left panel) but is expressed in podocytes of $\alpha 3^{--}$kidneys as shown by double labeling with synaptopodin (right panels). ratio: control, $26 \pm 12.78 ; 24$ hours' LPS, $205 \pm 150.01, P<0.01$; dipstick: control (trace $=0$ ), $0.0 \pm 0.21 ; 24$ hours' LPS, $2.0 \pm 0.71$, $P<0.001$. These results showed that changes in urinary protein detected with both tests were comparable.

Microbead experiments. The coating of microbeads was adapted from the method of Sedwick and coworkers (31). Sulfate-coated blue fluorescent microbeads $(6.2 \mu \mathrm{m}$ in diameter; Interfacial Dynamics Corp., Portland, Oregon, USA) were coated with polyclonal anti-B7-1 (16-10A1) antibody or polyclonal antidendrin (negative control). In addition, murine CTLA-4-Ig with a mutated Fc-binding site (32) or uncoated microbeads were used. Twenty microliters of beads per $5 \mu \mathrm{g}$ protein were used for surface coating. Podocytes growing in 24-well plates were incubated overnight with the coated beads, followed by immunocytochemistry.

\section{Results}

B7-1 is induced in podocytes in genetic and drug-induced models of FP effacement. Podocytes in kidneys of $\alpha 3^{-/-}$mice are unable to maintain normal podocyte structure, including the elaboration of mature FPs along the GBM (12). Therefore $\alpha 3^{-/-}$mice provide an excellent genetic model to study podocyte FP effacement. $\alpha 3^{-/-}$mice die during the perinatal period with severe kidney and lung defects, precluding extensive study of the role of this cell matrix receptor in podocytes in vivo. To delineate the molecular mechanisms underlying FP effacement in $\alpha 3^{-/-}$mice, we took advantage of our previously established cell culture system for WT podocytes (21) and generated $\alpha 3^{-/-}$conditionally immortalized podocyte cell lines. In an effort to identify genes critically involved in the development of FP effacement and proteinuria, we performed differential display PCR of total RNA harvested from cultured WT and $\alpha 3^{-/-}$podocytes. Three PCR products $\left(\mathrm{A}_{1-3}\right)$ were differentially expressed between WT and $\alpha 3^{-/-}$podocytes. Two clones, $\mathrm{A} 3_{1}$ (Figure $1 \mathrm{~A}$ ) and $\mathrm{A} 3_{2}$, were expressed by $\alpha 3^{-/-}$cells and one, $\mathrm{A} 3_{3}$, only by WT cells (data not shown). We confirmed the differential expression of all three clones by quantitative RT-PCR (24) (Figure 1B and data not shown). The expression of $\mathrm{A} 3_{1}$ was 14 -fold higher in $\alpha 3^{-/-}$than in
WT podocytes (Figure 1B) and therefore was evaluated in detail. The 400-bp product of $A 3_{1}$ was identical with a mouse myotube EST clone (AA673837). Using the 400-bp product as a probe, we isolated one clone from a mouse skeletal muscle cDNA library that covered the complete EST clone and extended 516 bp into exon 6 of the mouse B7-1 gene (33). The overlap with B7-1 ended at the $3^{\prime}$ end of exon 6 . Downstream from exon 6 was complete sequence diversion from the previously identified mouse B7-1 cDNA, suggesting the existence of an additional exon 7 forming a portion of the $3^{\prime}$ untranslated region (EMBL/GenBank accession no. AJ278965). The same novel splice variant of B7-1 was also cloned by RT-PCR from $\alpha 3^{-/-}$podocytes (data not shown). We then tested whether the restoration of $\alpha 3$ integrin expression would diminish B7-1 expression. Stable transfection of $\alpha 3^{-/-}$podocytes with human $\alpha 3$ integrin cDNA returned B7-1 expression to WT levels (Figure 1C). A control RT-PCR for the housekeeping gene GAPDH showed equal RNA concentrations (Figure 1C). Similar to the inactivation of the $\alpha 3$ integrin gene, a single injection of puromycin aminonucleoside (PAN) induces podocyte FP effacement and proteinuria in rats (34) and mice (35). In vitro, puromycin leads to the reorganization of the actin cytoskeleton, focal contacts, and SD proteins in podocytes (26, 36). The application of puromycin to WT podocytes for 48 hours caused a dose-dependent increase in B7-1 mRNA levels (Figure 1D). To test whether the effect of puromycin on B7-1 induction in WT cells was independent of $\alpha 3$ integrin expression, the mRNA expression of B7-1 and $\alpha 3$ integrin was analyzed in WT podocytes exposed to PAN for 6 and 24 hours. An induction of B7-1 mRNA expression could be observed, whereas the $\alpha 3$ integrin mRNA expression was not altered (Figure 1E). These studies showed that the induction of B7-1 can occur independent of changes in $\alpha 3$ integrin expression. To explore whether B7-1 was induced in $\alpha 3^{-/-}$podocytes in situ, we analyzed the expression of B7-1 in kidney sections of E18 embryonic WT and $\alpha 3^{-/-}$mice by immunofluorescence microscopy. No B7-1 labeling was found in WT kidneys (Figure 1F, left panel). In contrast, a strong expression of B7-1 was found in podocytes of $\alpha 3^{-/-}$kidneys as shown by double labeling with the podocyte marker 


\section{A}

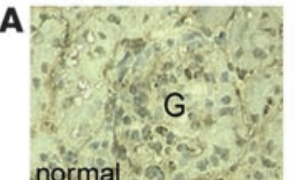

B $37-1$
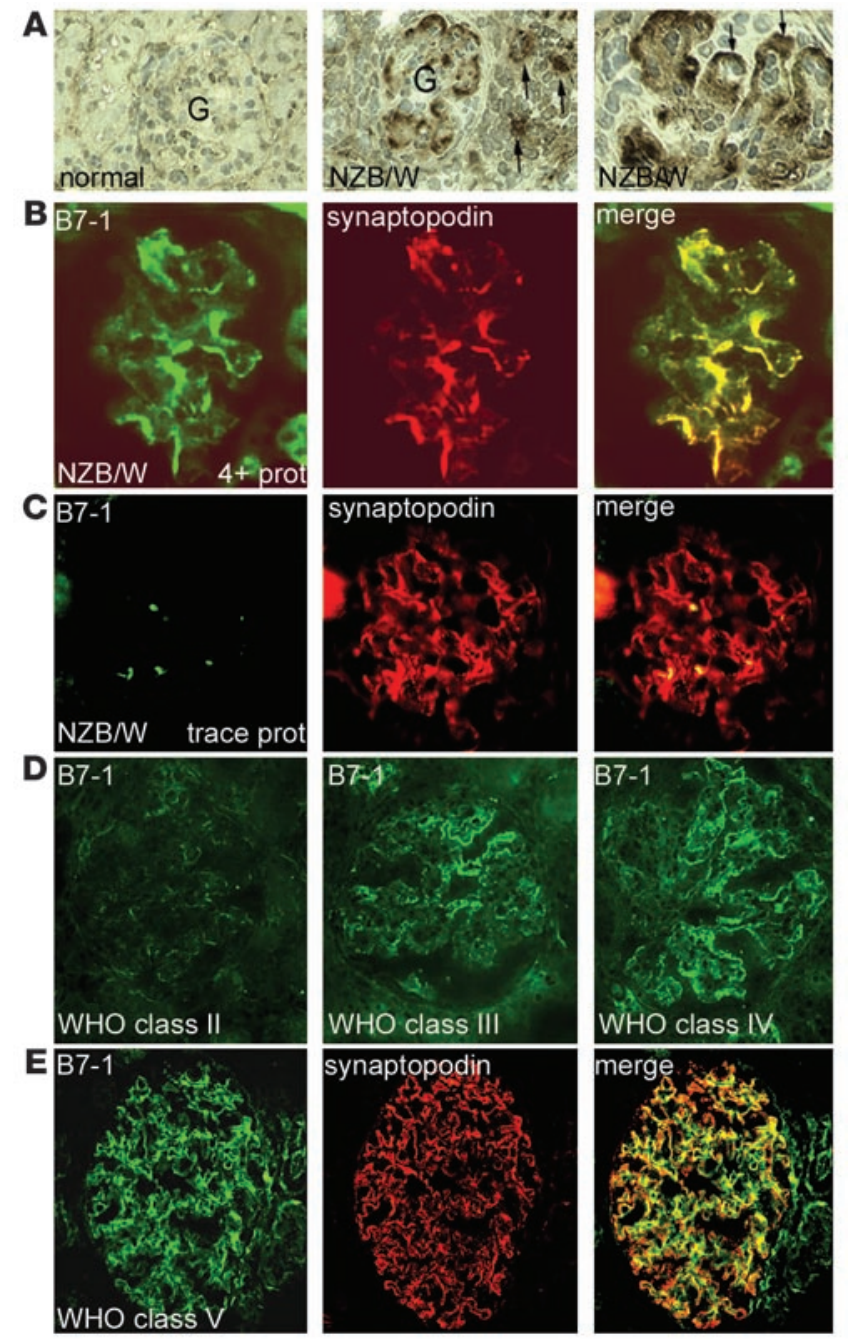

synaptopodin

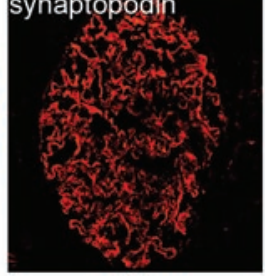

synaptopodin (25) (Figure 1F, right panels). In summary, the induction of B7-1 in both genetic and drug-induced models suggests that podocyte B7-1 expression can be regulated by various stimuli and may play a general role in different diseases with FP effacement and proteinuria.

Podocyte B7-1 expressed in human and murine lupus nephritis correlates with disease stage and severity of proteinuria. To explore the significance of B7-1 in podocytes in renal diseases with FP effacement and urinary protein loss, we analyzed B7-1 expression in New Zealand black/white (NZB/W) $\mathrm{F}_{1}$ mice, an established murine model of lupus glomerulonephritis (37). While no B7-1 expression was detected in WT kidneys (Figure 2A, left panel), a strong induction was noted in glomeruli of mice with active lupus glomerulonephritis (Figure 2A, middle panel). At higher magnification, $\mathrm{B} 7-1$ was detected in podocytes overlying glomerular capillaries (Figure 2A, right panel). In addition, as expected, B7-1 expression was found in interstitial lymphocytes (Figure 2A, middle panel, arrows). Double-labeling studies with synaptopodin (25) showed that glomerular B7-1 was expressed only in podocytes, not in mesangial cells or infiltrating cells (Figure 2B). Importantly, the expression of B7-1 in podocytes correlated with the severity of proteinuria, since B7-1 was strongly expressed in 30-week-old 4+ proteinuric lupus mice (Figure 2B) but not in

\section{Figure 2}

B7-1 expression in podocytes in murine and human lupus nephritis correlates with the severity of proteinuria. (A) B7-1 is not expressed in normal mouse kidney (left). In the lupus kidney, strong glomerular (G) B7-1 expression is observed (middle). In addition, infiltrating interstitial B cells stain positive (arrows). At higher magnification (right), B7-1 expression is found in podocytes (arrows) in the glomerulus. (B) Double labeling with synaptopodin confirmed that intraglomerular B7-1 expression is restricted to podocytes in 4+ proteinuric (4+ prot) nephritis. (C) B7-1 is absent in an age-matched lupus mouse with trace proteinuria (trace prot). ( $\mathbf{D}$ and $\mathbf{E}$ ) In human lupus nephritis, glomerular B7-1 expression increases from WHO class II to $\mathrm{V}$ and colocalizes with synaptopodin in podocytes (E).

age-matched animals that showed only trace proteinuria (Figure $2 \mathrm{C})$. To determine the significance of glomerular B7-1 expression in human glomerulonephritis, we analyzed B7-1 expression in human kidney biopsies from different stages of lupus nephritis. Glomerular B7-1 expression correlated with the disease stage in that it increased from WHO class II to $\mathrm{V}$ and was strongest in WHO class $\mathrm{V}$ kidney biopsies (Figure 2, D and $\mathrm{E}$ ). As in the murine condition (Figure 2C), glomerular B7-1 colocalized with the podocyte marker synaptopodin (25) (Figure 2E). Taken together, these data underscore the clinical relevance of $\mathrm{B} 7-1$ expression in podocytes. They suggest that B7-1 in podocytes contributes to the pathogenesis of proteinuria.

Induction of B7-1 in podocytes of nephrin ${ }^{-1-}$ mice. The nephrin gene NPHS1 is the target gene of congenital nephrotic syndrome of Finnish type, an autosomal-recessive disorder, characterized by massive proteinuria in utero and nephrosis at birth (3). Inactivation of NPHS1 in mice results in massive proteinuria, edema, and perinatal lethality $(27,38)$. To test whether the induction of $B 7-1$ in podocytes under nephrotic conditions is specific for lupus nephritis, we analyzed the expression of B7-1 in nephrin ${ }^{-/-}$mice (27). B7-1 colocalized with synaptopodin in podocytes of nephrin-- mice (Figure 3A). In contrast, no podocyte B7-1 expression was found in WT littermates (Figure 3B). These data imply that podocyte B7-1 expression is not specific for lupus nephritis but is also found in noninflammatory conditions with proteinuria and is not dependent on immune complexes.

B7-1 expression in podocytes in vitro is induced by LPS through TLR-4 signaling and leads to the reorganization of the actin cytoskeleton. B7-1 expression in APCs can be induced by LPS through TLR-4/CD14mediated signaling (20). Interestingly, podocytes constitutively express TLR-4 and its coreceptor CD14 and hence respond to LPS (Figure 4A). Therefore, we explored the effects of LPS on podocytes. Exposure to LPS specifically upregulated B7-1 (Figure $4 \mathrm{~B}$ ) and induced the disruption of stress fibers (Figure 4C), creating a cortical ring of $\mathrm{F}$-actin and a submembranous space lacking actin fibers (Figure 4D). This cytopathic effect is reminiscent of the actin changes that are observed in FP effacement in vivo (1) and suggests that LPS may contribute to the pathogenesis of FP effacement and proteinuria through the reorganization of the podocyte actin cytoskeleton.

LPS upregulates B7-1 in podocytes in vivo and leads to nephrotic syndrome in WT but not in B7-1-1- mice. To test this hypothesis further, we injected a single low dose of LPS intraperitoneally into adult female WT BALB/c mice and analyzed the effects on kidney histology, podocyte B7-1 expression, and urinary protein excretion. Histologically, kidneys of LPS-injected mice were 
B7-1
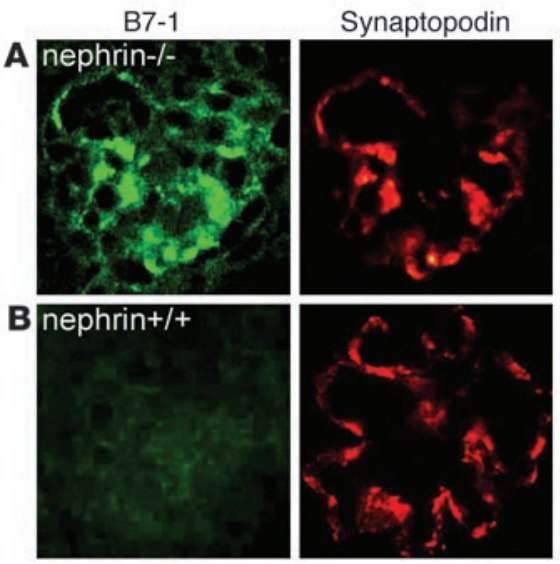
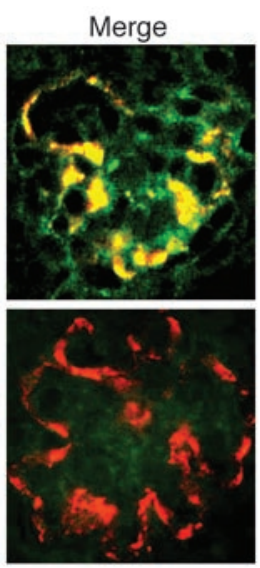

Figure 3

Induction of B7-1 in podocytes of nephrin ${ }^{-1-}$ mice. (A) B7-1 is induced in podocytes of nephrin ${ }^{-1}$ mice as shown by double labeling with synaptopodin. (B) B7-1 is absent from podocytes of WT littermate controls. These data establish that the expression of B7-1 in podocytes in vivo can occur independently of immune complexes or inflammatory cells.

not distinguishable from PBS-injected controls (Figure 5A). In particular, no signs of inflammation were found. LPS induced upregulation of B7-1 in podocytes (Figure 5B) and FP effacement within 24 hours (Figure 5D, left panel). In contrast, PBS injection did not induce podocyte B7-1 expression (Figure 5C) or FP effacement (Figure 5D, right panel). Most significantly, LPS injection caused severe proteinuria $(n=13$; base line, $0.8 \pm 0.4$, vs. LPS, $2.7 \pm 0.9 ; P<0.001$, two-sided $t$ test) within 24 hours (Figure $5 \mathrm{G}$ ). In contrast, no proteinuria was found in PBS-injected controls (data not shown). We next established that LPS-induced nephrotic syndrome is independent of the mouse strain, since we found it also in WT $129(n=9$; base line, $0.7 \pm 0.5$, vs. LPS, $2.1 \pm 0.78 ; P<0.001, t$ test $)$ and C57BL/ 6 mice $(n=23$; base line, $0.5 \pm 0.29$, vs. LPS, $1.9 \pm 0.56 ; P<0.0001, t$ test) (Figure $5 \mathrm{G}$ ). The LPS-induced proteinuria was transient and returned to base-line levels after 72 hours (data not shown). To establish a causal relationship between podocyte B7-1 upregulation and development of proteinuria, the experiments were repeated with $\mathrm{B} 7-1^{-/-}$mice (28). No proteinuria was observed in LPS-injected B7-1/- mice $(n=5$; base line, $1.0 \pm 0$, vs. LPS, $0.6 \pm 0.55$; $P$ not significant, $t$ test) (Figure 5G). The differences in proteinuria between WT and B7-1/- mice were statistically significant $(P<0.001, t$ test). These data suggest that B7-1 induction in podocytes is necessary for the development of LPS-induced proteinuria.

LPS-induced nephrotic syndrome is independent of T or B cells. To rule out that the LPS-induced proteinuria resulted from the activation of T or B cells rather than from a direct action on podocytes, SCID mice, which are devoid of T and B cells (29) were treated with LPS. As in WT mice, LPS injection in SCID mice caused podocyte B7-1 expression (Figure $5 \mathrm{E}$ ) and severe proteinuria ( $n=6$; base line, $1.2 \pm 0.4$, vs. LPS, $3.2 \pm 0.9 ; P<0.001$, $t$ test) within 24 hours (Figure $5 \mathrm{G}$ ). In contrast, B7-1 staining was absent from PBS-treated SCID mice (Figure 5F). Hence, the induction of B7-1 expression by podocytes in response to LPS is independent of T or $\mathrm{B}$ cells. Taken together, these data indicate a causal link between podocyte B7-1 expression and urinary protein loss that is independent of lymphocyte infiltration or activation.

B7-1 modifies the glomerular filter by sequestration of SD proteins. How does B7-1 upregulation in podocytes contribute to the pathogenesis of proteinuria? To test whether the ligation of B7-1 transduces intracellular signals in podocytes, thereby modulating the composition of the SD, we used a microbead approach to cross-link B7-1. With this approach, three key proteins of the SD, nephrin (3), CD2AP (4), and ZO-1 (39), were analyzed in $\alpha 3^{-/}$ podocytes by immunocytochemistry after binding of microbeads coated with CTLA-4-Ig or anti-B7-1 antibody (Figure 6). The detailed microscopic analysis revealed that nephrin was redistributed from cell-cell contacts (Figure 6A) and accumulated around CTLA-4-Ig-coated (Figure 6B) or anti-B7-1-coated microbeads (Figure 6C). The association of SD proteins with anti-B7-1-coated microbeads was confirmed by confocal microscopy, which showed a ring of nephrin lining the microbeads (Figure 6D). Similar results were obtained for CD2AP, which was redistributed from the cell membrane and the actin filaments (Figure 6E) to microbeads (Figure 6, F-H). ZO-1 relocated from cell-cell junctions (Figure 6I) to the microbeads (Figure 6, J-L). To show that the redistribution of SD proteins was specifically mediated by B7-1, several control experiments were performed. First, microbeads were coated with a mutated variant of murine CTLA-4-Ig that can no longer bind to Fc receptor. Second, microbeads were coated with an irrelevant antibody with an intact Fc fragment. The microbeads coated with the irrelevant antibody (dendrin) could to some extent adhere to podocytes but did not cause the rearrangement of ZO-1 (Figure 6M). Third, uncoated microbeads were used. No binding was observed with uncoated microbeads or beads coated with the irrelevant antibody (data not shown). Moreover, B7-1 clustering beads had no effect on cultured WT podocytes, which do not express B7-1 (data not shown). In summary, the results of the bead experiments are specific and conclusive. They suggest that B7-1 may contribute to the pathogenesis of proteinuria by disrupting the SD complex.

\section{Discussion}

This study is the first to demonstrate that B7-1 is expressed in podocytes under a variety of stress conditions. Moreover, the results of this study establish the clinical significance of

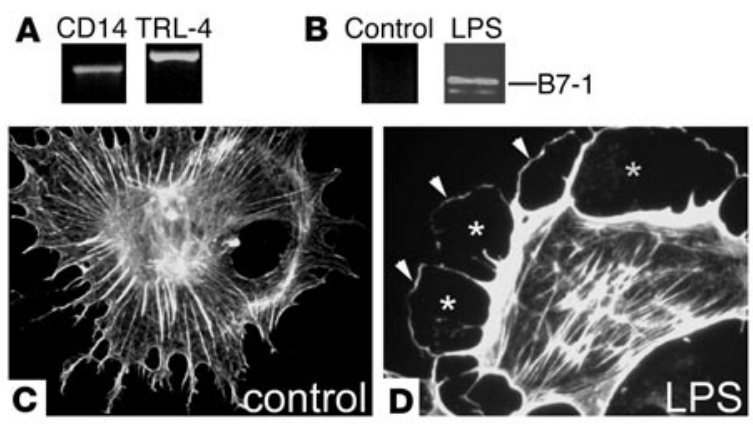

\section{Figure 4}

LPS-induced upregulation of B7-1 and actin cytoskeleton reorganization in podocytes in vitro. (A) Cultured podocytes express the LPS receptors CD14 and TLR-4 as shown by RT-PCR. (B) LPS treatment upregulates B7-1 mRNA expression and reorganizes the podocyte cytoskeleton from a stress fiber pattern (C), creating a cortical ring of F-actin (D; arrowheads) and a submembranous space devoid of actin fibers (asterisks). 
A

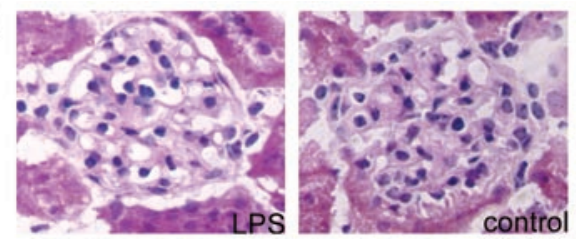

B7-1

B

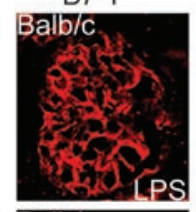

C

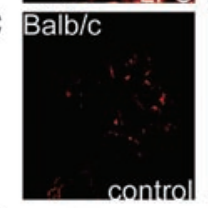
Synaptopodin
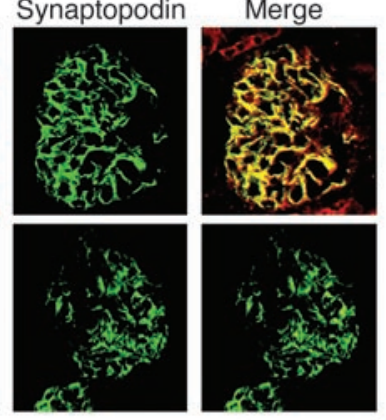

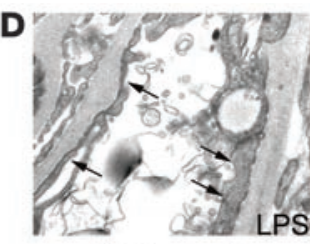

B7-1
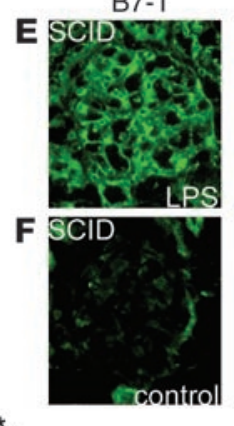

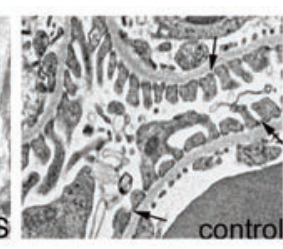

Synaptopodin
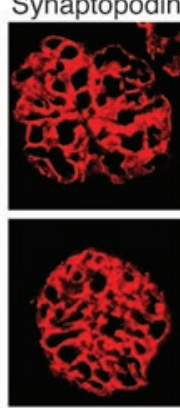

Merge
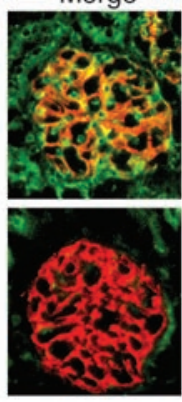

G

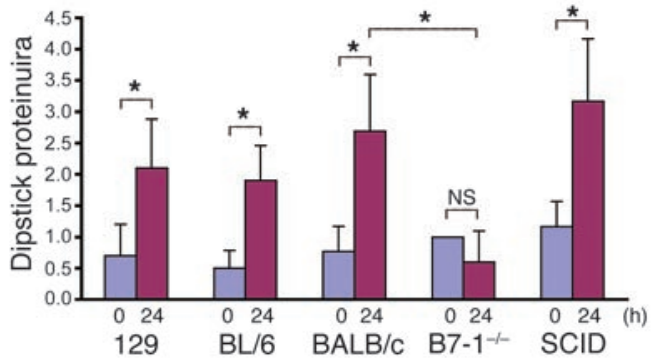

podocyte B7-1 expression and elucidate its role in the pathogenesis of nephrotic syndrome. We specifically demonstrate that podocyte B7-1 reorganizes the actin cytoskeleton of podocytes and modulates SD organization. The effects are independent of $\mathrm{T}$ cells and $\mathrm{B}$ cells and represent a novel unique function of $\mathrm{B} 7-1$. This study also is the first to show that LPS injection in mice induces B7-1-dependent nephrotic syndrome with FP effacement and urinary protein loss.

So far, B7-1 had been assumed to be predominantly expressed on B cells and other professional APCs. The traditional role of B7-1 is $\mathrm{T}$ cell costimulation (13-15). Along with the interaction of the $\mathrm{T}$ cell receptor with antigen-MHC complexes, the interaction of B7 with its receptors CD28 and CTLA- 4 on T cells plays a pivotal role in $\mathrm{T}$ cell-mediated immunity (13-15). In T cells, binding of $\mathrm{B} 7-1$ to its receptors on the $\mathrm{T}$ cell surface triggers migration of protein kinases, actin-binding proteins, and other accessory molecules to the T cell-APC interface $(40,41)$. This formation of the immunological synapse causes a rearrangement of the $\mathrm{T}$ cell actin cytoskeleton $(40,42-44)$, activates protein tyrosine kinases that couple to PI3K, and controls the activity of Rasand Rho-family GTPases in T cells (45).

The role of B7-1 in T cell costimulation is well established and defines a dialogue between a B7-1-expressing APC and a T cell to which the costimulatory signal is conveyed (13-15). In contrast, B7-1-mediated outside-in signaling in B7-1-expressing APCs is still obscure. With respect to outside-in signaling through B7-1 on B7-1-expressing cells, two studies have been published. One study showed that cross-linking of B7-1 on B lymphoma Raji cells induces tyrosine phosphorylation of intracellular proteins, indicating a role of B7-1 in outside-in signaling (46). Further support for a role of B7-1 in outside-in signaling comes from a recent report showing that CTLA-4-Ig-mediated ligation of B7-1 regulates tryptophan catabolism in dendritic cells (47). These changes are reminiscent of the changes seen in SD protein redistribution after ligation/activation of B7-1 in podocytes (Figure 6).

In human keratinocytes, B7-1 gene expression is induced by allergens and irritants (16). In P815 mastocytoma tumor cells, B7-1 gene expression is induced by oxidative stress (17). In APCs, MyD88-independent LPS/TLR-4 signaling is the strongest activator of B7-1 expression $(20,48)$. Similarly, B7-1 induction in podocytes appears to be downstream of various signaling pathways, including TLR signaling (Figure 5). Generally, somatic cells grown in culture are, for the most part, quite indifferent to LPS (20), and earlier studies had shown that macrophages are the primary target cells of LPS in septic shock (20). Here we demonstrate that podocytes constitutively express the LPS receptor TLR-4 and its coreceptor CD14 and respond to LPS exposure with upregulation of B7-1. This is another unanticipated finding of the present study.

The LPS experiments (Figures 4 and 5) allowed us to explore several important questions. First, they established that the induction of podocyte B7-1 in conditions with FP effacement and proteinuria was causative and not the consequence of the nephrotic condition, since B7-1/- mice were protected from LPSinduced proteinuria. Second, one might argue that the effects of LPS are predominantly mediated through the action of $\mathrm{T}$ cells. Our data refute this hypothesis, because (a) SCID mice lacking $\mathrm{T}$ and $\mathrm{B}$ cells showed the same degree of FP effacement and proteinuria as WT mice; (b) exposure of cultured podocytes in the absence of any other cells was sufficient to upregulate B7-1 and reorganize the podocyte actin cytoskeleton; and (c) the cell-free microbead-mediated cross-linking of B $7-1$ on $\alpha 3^{-/-}$podocytes was 


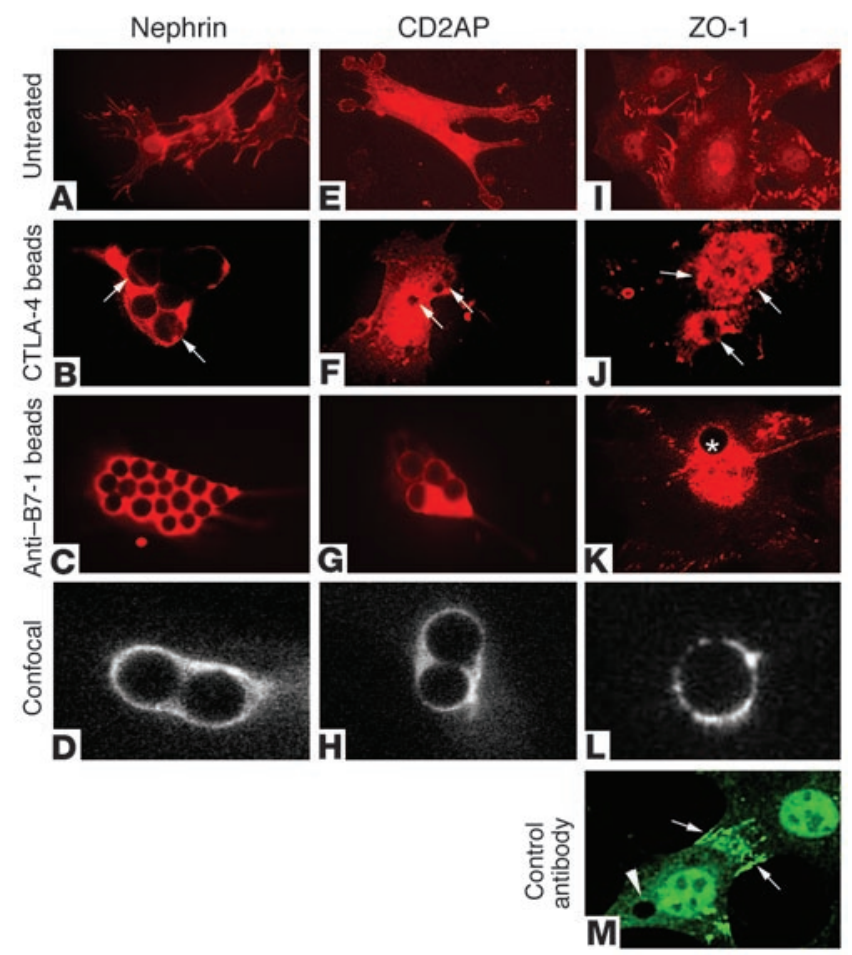

sufficient to redistribute vital SD proteins. Collectively, these data suggest that B7-1 expression by podocytes represents a pathogenic mechanism for disruption of the glomerular filtration apparatus that is independent of $\mathrm{T}$ cell activation.

An intriguing question remains, why do podocytes upregulate B7-1? In the grass shrimp, Palaemonetes pugio, podocytes are involved in the ultrafiltration of hemolymph where micro- and macromolecular substances are cleared (49). In the pre-molt stage of this shrimp, podocyte assemblages extend into the efferent hemolymph channels and are in direct contact with immune cells, and they may serve as a component of the LPS-sensing (20) innate immune response. The upregulation of podocyte B7-1 by LPS may be a phylogenetically preserved mechanism, which can be reactivated under pathological conditions, e.g., in patients with Gram-negative sepsis. Consistent with this idea, the development of transient proteinuria has been found during the course of Gram-negative sepsis (50). Moreover, a prospective study showed that in postoperative septic patients microalbuminuria is an early indicator of increased glomerular permeability, but not in nonseptic patients (51). The resulting, transient, proteinuria may be a physiological response aimed at clearing the circulation from pathogen-associated molecules. In human minimal-change disease, the onset of nephrotic syndrome is often preceded by an infection or allergic reaction (52). This raises the intriguing possibility that nephrotic syndrome in minimal-change disease represents the persistence of a normally beneficial response caused by genetic defects in the B7-1 pathway or by other unphysiological activators. The sustained B7-1 activation in podocytes, in turn, results in continuing nephrotic syndrome.

In summary, we have demonstrated a novel function for B7-1 in the pathogenesis of proteinuria that is distinct from its role in costimulation (Figure 7). Our findings suggest a novel function for B7-1 in danger signaling by podocytes. The B7-1 knockout by itself is not conclusive since it is not podocyte specific. While the

\section{Figure 6}

Redistribution of SD proteins to sites of B7-1 engagement. (A) In untreated $\alpha 3^{-/-}$podocytes, nephrin is expressed at cell-cell contacts. (B and C) Incubation of podocytes with microbeads coated with CTLAIg (B) or anti-B7-1 antibody (C) redistributes nephrin around microbeads (arrows). (D) The association of nephrin with microbeads was confirmed by confocal microscopy. (E-L) Similar redistribution patterns were seen for CD2AP $(\mathbf{E}-\mathbf{H})$ and ZO-1 (I-L). Arrows in $\mathbf{F}$ and $\mathbf{J}$ : accumulation of CD2AP (F) and ZO-1 (J) around microbeads. Asterisk in K: microbead. (M) In contrast, microbeads coated with an irrelevant antibody could to some extent adhere to podocytes (arrowhead) but did not affect the localization of ZO-1 at cell-cell contacts (arrows).

SCID mice control for T or B cell-mediated effects, it is still possible that dendritic cells or other APCs might contribute to the observed effects. Future studies with a podocyte-specific B7-1 knockout should allow us to address this question.

We propose that transient B7-1-induced nephrotic syndrome may be a physiological response. Clearly, future studies will be required to explore this hypothesis in detail, but it could potentially explain how a normally beneficial danger-sensing mechanism can have deleterious effects in certain genetic backgrounds. Understanding the role of B7-1 in podocytes should allow new avenues toward the development of novel, selective podocyte-protective therapies that modulate danger signaling in podocytes and tackle proteinuria. It will be interesting to see whether B7-1-dependent pathways are also operative in chronic kidney disease.

\section{Acknowledgments}

We thank Torsten Heider for technical assistance and Michael Cammer (Albert Einstein College of Medicine) for help with confocal microscopy. We thank Andrey S. Shaw for anti-CD2AP and Lawrence B. Holzman for anti-nephrin antibodies and Amin Arnaout for helpful discussion and critical reading of the manu-

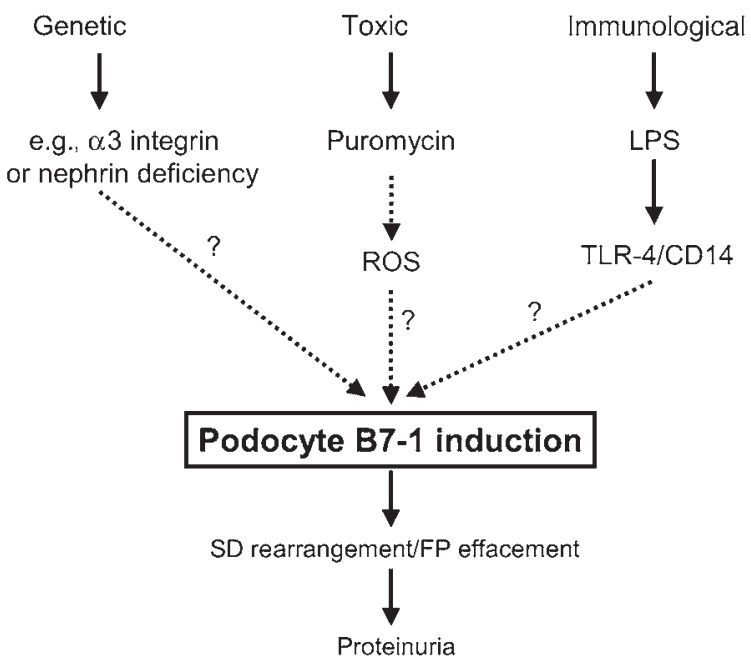

Figure 7

Pathways leading to B7-1-mediated proteinuria. Various stimuli may lead to podocyte B7-1 induction. These include genetic stimuli (e.g., deletion of $\alpha 3$ integrin or nephrin), toxic stimuli (PAN-induced reactive oxygen species [ROS]), or direct stimulation of the TLR-4/CD14 receptor on the podocyte. B7-1 then induces FP effacement and disruption of the SD complex, thereby modifying glomerular permselectivity. 
script. Jochen Reiser was a fellow of the Graduiertenkolleg Experimentelle Nieren- und Kreislaufforschung of the Deutsche Forschungsgemeinschaft (DFG) and Martin Loos of the Deutscher Akademischer Austauschdienst. Gero von Gersdorff is supported by a fellowship from the Lupus Foundation of New York, and Karin Schwarz and Katsuhiko Asanuma by the Kidney and Urology Foundation of America (KUFA). Jun Oh was supported by the DFG and by KUFA. This work was supported by grants from the Lupus Research Institute, the NIH (DK57683 and DK062472), and the George M. O'Brien Kidney Center (DK064236) to Peter Mundel, as well as NIH grant DK55001 to Raghu Kalluri.
Received for publication October 28, 2003, and accepted in revised form March 23, 2004.

Address correspondence to: Peter Mundel, Division of Nephrology, Albert Einstein College of Medicine, 1300 Morris Park Avenue, New York, New York 10461, USA. Phone: (718) 430-3219; Fax: (718) 430-8963; E-mail: mundel@aecom.yu.edu.

Jochen Reiser's present address is: Renal Unit, Massachusetts General Hospital, and Harvard Medical School, Charlestown, Massachusetts, USA.
1. Somlo, S., and Mundel, P. 2000. Getting a foothold in nephrotic syndrome. Nat. Genet. 24:333-335.

2. Kriz, W., Gretz, N., and Lemley, K.V. 1998. Progression of glomerular diseases: is the podocyte the culprit? Kidney Int. 54:687-697.

3. Kestila, M., et al. 1998. Positionally cloned gene for a novel glomerular protein-nephrin-is mutated in congenital nephrotic syndrome. Mol. Cell. 1:575-582.

4. Shih, N.Y., et al. 1999. Congenital nephrotic syndrome in mice lacking CD2-associated protein. Science. 286:312-315.

5. Kaplan, J.M., et al. 2000. Mutations in ACTN4, encoding alpha-actinin-4, cause familial focal segmental glomerulosclerosis. Nat. Genet. 24:251-256.

6. Boute, N., et al. 2000. NPHS2, encoding the glomerular protein podocin, is mutated in autosomal recessive steroid-resistant nephrotic syndrome. Nat. Genet. 24:349-354.

7. Donoviel, D.B., et al. 2001. Proteinuria and perinatal lethality in mice lacking neph1, a novel protein with homology to nephrin. Mol. Cell. Biol. 21:4829-4836.

8. Adler, S. 1992. Characterization of glomerular epithelial cell matrix receptors. Am. J. Pathol. 141:571-578.

9. Regele, H.M., et al. 2000. Glomerular expression of dystroglycans is reduced in minimal change nephrosis but not in focal segmental glomerulosclerosis. J. Am. Soc. Nephrol. 11:403-412.

10. Raats, C.J., et al. 2000. Expression of agrin, dystroglycan, and utrophin in normal renal tissue and in experimental glomerulopathies. Am.J. Pathol. 156:1749-1765.

11. Kreidberg, J.A. 2000. Functions of alpha3beta1 integrin. Curr. Opin. Cell Biol. 12:548-553.

12. Kreidberg, J.A., et al. 1996. Alpha 3 beta 1 integrin has a crucial role in kidney and lung organogenesis. Development. 122:3537-3547.

13. Abbas, A.K., and Sharpe, A.H. 1999. T-cell stimulation: an abundance of B7s. Nat. Med. 5:1345-1346.

14. Henry, J., Miller, M.M., and Pontarotti, P. 1999. Structure and evolution of the extended B7 family. Immunol. Today. 20:285-288.

15. Chambers, C.A., and Allison, J.P. 1999. Costimulatory regulation of $\mathrm{T}$ cell function. Curr. Opin. Cell Biol. 11:203-210.

16. Wakem, P., et al. 2000. Allergens and irritants transcriptionally upregulate CD80 gene expression in human keratinocytes. J. Invest. Dermatol. 114:1085-1092.

17. Donepudi, M., Raychaudhuri, P., Bluestone, J.A., and Mokyr, M.B. 2001. Mechanism of melphalaninduced B7-1 gene expression in P815 tumor cells. J. Immunol. 166:6491-6499.

18. Satoh, S., et al. 2002. Glomerular endothelium exhibits enhanced expression of costimulatory adhesion molecules, CD80 and CD86, by warm ischemia/reperfusion injury in rats. Lab. Invest. 82:1209-1217.

19. Takeda, K., Kaisho, T., and Akira, S. 2003. Toll-like receptors. Annu. Rev. Immunol. 21:335-376.
20. Beutler, B., and Rietschel, E.T. 2003. Innate immune sensing and its roots: the story of endotoxin. Nat. Rev. Immunol. 3:169-176.

21. Mundel, P., et al. 1997. Rearrangements of the cytoskeleton and cell contacts induce process formation during differentiation of conditionally immortalized mouse podocyte cell lines. Exp. Cell Res. 236:248-258.

22. Jat, P.S., et al. 1991. Direct derivation of conditionally immortal cell lines from an $\mathrm{H}-2 \mathrm{~Kb}-\mathrm{tsA} 58$ transgenic mouse. Proc. Natl. Acad. Sci. U. S. A. 88:5096-5100.

23. Wang, Z., et al. 1999. $\alpha 3 \beta 1$ integrin regulates epithelial cytoskeletal organization. J. Cell Sci. 112:2925-2935.

24. Kretzler, M., et al. 1996. Novel mouse embryonic renal marker gene products differentially expressed during kidney development. Am. J. Physiol. 271:F770-F777.

25. Mundel, P., et al. 1997. Synaptopodin: an actinassociated protein in telencephalic dendrites and renal podocytes. J. Cell Biol. 139:193-204.

26. Reiser, J., et al. 2000. Regulation of mouse podocyte process dynamics by protein tyrosine phosphatases. Kidney Int. 57:2035-2042.

27. Hamano, Y., et al. 2002. Determinants of vascular permeability in the kidney glomerulus. J. Biol. Chem. 277:31154-31162.

28. Borriello, F., et al. 1997. B7-1 and B7-2 have overlapping, critical roles in immunoglobulin class switching and germinal center formation. Immunity. 6:303-313.

29. Bosma, G.C., Custer, R.P., and Bosma, M.J. 1983. A severe combined immunodeficiency mutation in the mouse. Nature. 301:527-530.

30. Barisoni, L., Kriz, W., Mundel, P., and D’Agati, V. 1999. The dysregulated podocyte phenotype: a novel concept in the pathogenesis of collapsing idiopathic focal segmental glomerulosclerosis and HIV-associated nephropathy. J. Am. Soc. Nephrol. 10:51-61.

31. Sedwick, C.E., et al. 1999. TCR, LFA-1, and CD28 play unique and complementary roles in signaling $\mathrm{T}$ cell cytoskeletal reorganization. J. Immunol. 162:1367-1375.

32. Mihara, M., et al. 2000. CTLA4Ig inhibits T celldependent B-cell maturation in murine systemic lupus erythematosus. J. Clin. Invest. 106:91-101.

33. Borriello, F., et al. 1994. Characterization of the murine B7-1 genomic locus reveals an additional exon encoding an alternative cytoplasmic domain and a chromosomal location of chromosome 16, band B5. J. Immunol. 153:5038-5048.

34. Whiteside, C., Prutis, K., Cameron, R., and Thompson, J. 1989. Glomerular epithelial detachment, not reduced charge density, correlates with proteinuria in adriamycin and puromycin nephrosis. Lab. Invest. 61:650-660.

35. Cheng, Z.Z., et al. 2003. Hypercholesterolemia is a prerequisite for puromycin inducible damage in mouse kidney. Kidney Int. 63:107-112.

36. Saleem, M.A., et al. 2002. Co-localization of nephrin, podocin, and the actin cytoskeleton: evidence for a role in podocyte foot process formation. Am. J. Pathol. 161:1459-1466.

37. Kelley, V.E., and Cavallo, T. 1976. An ultrastructural study of the glomerular slit diaphragm in New Zealand black/white mice. Lab. Invest. 35:213-220.

38. Putaala, H., Soininen, R., Kilpelainen, P., Wartiovaara, J., and Tryggvason, K. 2001. The murine nephrin gene is specifically expressed in kidney, brain and pancreas: inactivation of the gene leads to massive proteinuria and neonatal death. Hum. Mol. Genet. 10:1-8.

39. Schnabel, E., Anderson, J.M., and Farquhar, M.G. 1990. The tight junction protein ZO-1 is concentrated along slit diaphragms of the glomerular epithelium. J. Cell Biol. 111:1255-1263.

40. Tamir, A., Eisenbraun, M.D., Garcia, G.G., and Miller, R.A. 2000. Age-dependent alterations in the assembly of signal transduction complexes at the site of T cell/APC interaction. J. Immunol. 165:1243-1251.

41. Wulfing, C., and Davis, M.M. 1998. A receptor/ cytoskeletal movement triggered by costimulation during T cell activation. Science. 282:2266-2269.

42. Bromley, S.K., et al. 2001. The immunological synapse. Annu. Rev. Immunol. 19:375-396.

43. Shaw, A.S., and Allen, P.M. 2001. Kissing cousins: immunological and neurological synapses. Nat. Immunol. 2:575-576.

44. Grakoui, A., et al. 1999. The immunological synapse: a molecular machine controlling $T$ cell activation. Science. 285:221-227.

45. Acuto, O., and Cantrell, D. 2000. T cell activation and the cytoskeleton. Annu. Rev. Immunol. 18:165-184.

46. Hirokawa, M., Kuroki, J., Kitabayashi, A., and Miura, A.B. 1996. Transmembrane signaling through CD80 (B7-1) induces growth arrest and cell spreading of human B lymphocytes accompanied by protein tyrosine phosphorylation. Immunol. Lett. 50:95-98.

47. Grohmann, U., et al. 2002. CTLA-4-Ig regulates tryptophan catabolism in vivo. Nat. Immunol. 3:1097-1101.

48. Kaisho, T., Takeuchi, O., Kawai, T., Hoshino, K., and Akira, S. 2001. Endotoxin-induced maturation of MyD88-deficient dendritic cells. J. Immunol. 166:5688-5694.

49. Doughtie, D.G., and Rao, K.R. 1981. The syncytial nature and phagocytic activity of the branchial podocytes in the grass shrimp, Palaemonetes pugio. Tissue Cell. 13:93-104.

50. Pacquement, H., et al. 1989. Nephrotic syndrome and B leukemia [In French]. Arch. Fr. Pediatr. 46:741-742.

51. De Gaudio, A.R., Adembri, C., Grechi, S., and Novelli, G.P. 2000. Microalbuminuria as an early index of impairment of glomerular permeability in postoperative septic patients. Intensive Care Med. 26:1364-1368.

52. Eddy, A.A., and Symons, J.M. 2003. Nephrotic syndrome in childhood. Lancet. 362:629-639. 